\title{
Use of ganciclovir in the treatment of acquired cytomegalovirus disease in a preterm infant
}

\author{
H AMIN, MBBS, MRCP(UK), FRCPC, T JADAVJI, MD, FRCPC, FAAP,
} R SAUVE, MD, FRCPC, J GILL, MB, CHB, FRCPC

\begin{abstract}
The first use of ganciclovir in a preterm infant is reported. The 27 week appropriate-for-gestational-age male infant developed a disseminated cytomegalovirus infection subsequent to a blood transfusion. A daily dose of $10 \mathrm{mg} / \mathrm{kg}$ administered intravenously in two divided doses for a total of 14 days was
\end{abstract}

given without adverse clinical or toxic effects. The patient has remained well following discharge from hospital. Can J Infect Dis 1990;1(1):28-30

Key Words: Cytomegalovirus disease, Ganciclovir, Infants
$\mathrm{T}$ HE ADMINISTRATION OF CYTOMEGALOVIRUS (CMV) seropositive blood products to neonates in the perinatal period has been associated with the development of cytomegalovirus disease which may be fatal in up to $20 \%$ of infants $(1,2)$. Preterm infants weighing less than $2000 \mathrm{~g}$ are at the highest risk for complications from post natally acquired CMV infection $(3,4)$. The serious or fatal problems described include pneumonia, hepatitis, hemolytic anemia and long term neurological sequelae $(3,5)$. Recent studies have shown that the

Departments of Pediatrics, Microbiology and Infectious Disease. Foothills Hospital, The University of Calgary. Alberta Correspondence and reprints: Dr T Jadavji. Alberta Children's Hospital, 1820 Richmond Road SW. Calgary. Alberta T2T 5C7. Telephone (403) 229-7813

Received for publication January 23, 1990. Accepted April 19. 1990 drug ganciclovir (9-[1,3-dihyroxy-2-propoxymethyl] guanine, DHPG) is effective in the treatment of serious CMV infections in immunocompromised hosts (6-8). The successful use of ganciclovir is reported in a preterm infant who developed a disseminated CMV infection subsequent to blood transfusion. This is the first reported use of ganciclovir in a neonate.

\section{CASE REPORT}

A 27 week appropriate-for-gestational-age male infant weighing $1010 \mathrm{~g}$ was born by spontaneous vaginal delivery to a 23-year-old mother. Apgar scores were six at $1 \mathrm{~min}$ and seven at $5 \mathrm{mins}$. The neonate developed hyaline membrane disease and required assisted ventilation for 15 days and supplemental oxygen for a further 24 days. Subsequent progress was complicated by the 
development of bronchopulmonary dysplasia and retinopathy of prematurity. From day 46 onward, the patient became increasingly lethargic and developed an increasing number of apneic attacks with bradycardias and an increasing oxygen requirement. He was subsequently found to be hypotonic with marked hepatosplenomegaly. Following a full work-up for sepsis the patient was started on a course of broad spectrum antibiotics; however, he continued to deteriorate, and by day 56 required reintubation and assisted ventilation. Generalized petechiae over the skin, and recurrence of jaundice with mild direct (conjugated) hyperbilirubinemia, proteinuria and hematuria were noted. His platelet count was $59 \times 10^{9} / \mathrm{L}$ with a hematocrit of $0.42 \mathrm{~L} / \mathrm{L}(42 \%)$, reticulocyte count of $126 \times 10^{9} / \mathrm{L}(3.8 / 100 \mathrm{RBC})$ and fibrin degradation products 40 to $80 \mathrm{mg} / \mathrm{L}$. Direct and indirect Coombs' tests were negative. Blood, cerebrospinal fluid and urine cultures were negative and the antibiotics were stopped after $48 \mathrm{~h}$. During these first eight weeks of life, the patient had received six transfusions of packed red blood cells from different donors to replace blood lost, in order to maintain a hematocrit greater than $0.40 \mathrm{~L} / \mathrm{L}$ (40\%).

Ophthalmological assessment on day 60 revealed the presence of chorioretinitis. Urine samples taken on days 55 and 60 demonstrated a positive cytopathic effect from CMV. The presence of CMV in the urine, associated with hepatosplenomegaly, thrombocytopenia, pneumonia. and chorioretinitis, confirmed a clinical diagnosis of disseminated CMV disease. One transfusion of red blood cells that the patient received at day 9 was found in retrospect to be CMV antibody-positive. Normally all blood administered to babies in this unit is prescreened to be CMV antibody-negative. In view of the patient's critical state informed written parental consent was obtained for treatment with ganciclovir. A daily dose of $10 \mathrm{mg} / \mathrm{kg}$ administered intravenously in two divided doses was started on day 68. Further ophthalmological examination on day 74 revealed stage III retinopathy of prematurity, with no chorioretinitis. Ganciclovir was discontinued after a total of 14 days of treatment. There were no adverse clinical or toxic effects while on ganciclovir. The patient was discharged home on day 87 off oxygen, weighing $2600 \mathrm{~g}$ (10th percentile) with a length of $46 \mathrm{~cm}$ (10th percentile) and a head circumference of 33.3 $\mathrm{cm}$ (40th percentile). His chest was clear to auscultation with the liver palpable $4 \mathrm{~cm}$ below the right costal margin, and spleen palpable $3 \mathrm{~cm}$ below the left costal margin. Examination of the central nervous system was normal. No significant side effects from ganciclovir were observed, other than transient mild elevation of liver enzymes. Leukopenia, feeding intolerance, gastrointestinal hemorrhage and hypotension did not occur. No further blood transfusions were required. Pre- and post treatment immunoglobulin levels were normal. CMV antibody status was negative at the start of ganciclovir treatment, but later he became positive to CMV by both IgG and IgM ELISA. Urine cultures were negative three weeks after the course of ganciclovir and remained negative for $\mathrm{CMV}$ at four and six month follow-up examinations.

The patient has remained well following discharge from hospital, and follow-up at a chronological age of seven months with an adjusted age of four months showed his weight and length to be on the fifth percentile (weight $5 \mathrm{~kg}$, length $59 \mathrm{~cm}$ ) and head circumference on the 40th percentile $(41 \mathrm{~cm})$. Mild chest wall retractions with expiratory rhonchi on auscultation were felt to be compatible with the sequelae of bronchopulmonary dysplasia. His abdomen was protuberant with the liver palpable $1 \mathrm{~cm}$ below the right costal margin. There was no splenomegaly. The only abnormalities on neurological exam were bilateral exaggeration of his popliteal angles to between $120^{\circ}$ and $150^{\circ}$ with bilaterally exaggerated knee jerks and inconstant clonus of the left ankle. His gross motor function was at a four to five month level and fine motor function at a three month level. Ophthalmological exam showed no evidence of chorioretinitis. Auditory brain response testing confirmed that hearing in both ears was normal.

\section{DISCUSSION}

This patient developed symptomatic CMV infection with hepatosplenomegaly, thrombocytopenia, petechiae, jaundice with mild direct (conjugated) hyperbilirubinemia, pneumonia and chorioretinitis following a CMV antibody-positive transfusion. After 14 days of ganciclovir, no persistence of CMV infection was found. During therapy, no significant side effects other than transiently elevated liver function tests were observed. As this patient is at high risk for developmental, sensory. cognitive and intellectual deficits $(5,9,10)$, further long term follow-up will determine whether treatment with ganciclovir has altered or modified the patient's high risk category. The good clinical response and rapid improvement in ophthalmological findings are encouraging, and suggest that further clinical studies using ganciclovir to treat acquired CMV disease need to be undertaken to determine its pharmacokinetics, efficacy and benefit in the neonatal and immediate post natal period. 


\section{REFERENCES}

1. Yeager AS. Transfusion-acquired cytomegalovirus infection in newborn infants. Am J Dis Child 1974:128:478-83.

2. Yeager AS, Grumet FG, Hafleigh EB, et al. Prevention of transfusion-acquired cytomegalovirus infections in newborn infants. J Pediatr 1981;98:281-7.

3. Paryani S, Yeager AS, Hosford-Dunn H, et al. Sequelae of acquired cytomegalovirus infection in premature and sick term infants. J Pediatr 1985; 107:451-6.

4. Yeager AS, Palumbo PE, Malachowski N, et al. Sequelae of maternally derived cytomegalovirus infections in premature infants. J Pediatr 1983:102:918-22.

5. Conboy TJ, Pass RF, Stagno S, et al. Early clinical manifestations and intellectual outcome in children with symptomatic congenital cytomegalovirus infection. J Pediatr 1987;111:343-8.

6. Collaborative DHPG Treatment Study Group. Treatment of serious cytomegalovirus infections with 9- (1.3-dihydroxy-2-propoxymethyl) guanine in patients with AIDS and other immunodeficiencies. N Engl J Med 1986:314:801-5.

7. Chachova A. Dietefich D, Krasinski K. et al. 9-(1.3-dihydroxy-2-propoxymethyl) guanine (ganciclovir) in the treatment of cytomegalovirus gastrointestinal disease with the acquired immunodeficiency syndrome. Ann Intern Med 1987; 107:133-8.

8. Hecht DW, Snydman DR, Crumpacher CS, et al. Ganciclovir for the treatment of renal transplant associated primary cytomegalovirus pneumonia. $J$ Infect Dis 1988;157:187-90.

9. McCraken GH, Shinefield HR, Cobb K, et al. Congenital cytomegalic inclusion disease: A longitudinal study of 20 patients. Am J Dis Child 1969:1 17:522-39.

10. Pass RF, Stagno S, Myers GJ, et al. Outcome of symptomatic congenital cytomegalovirus infection: Results of long-term longitudinal follow-up. Pediatrics 1980;66:758-66. 


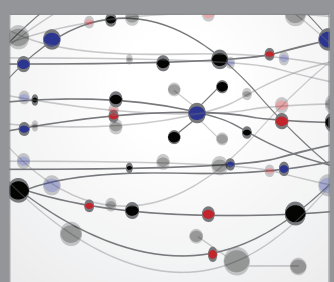

The Scientific World Journal
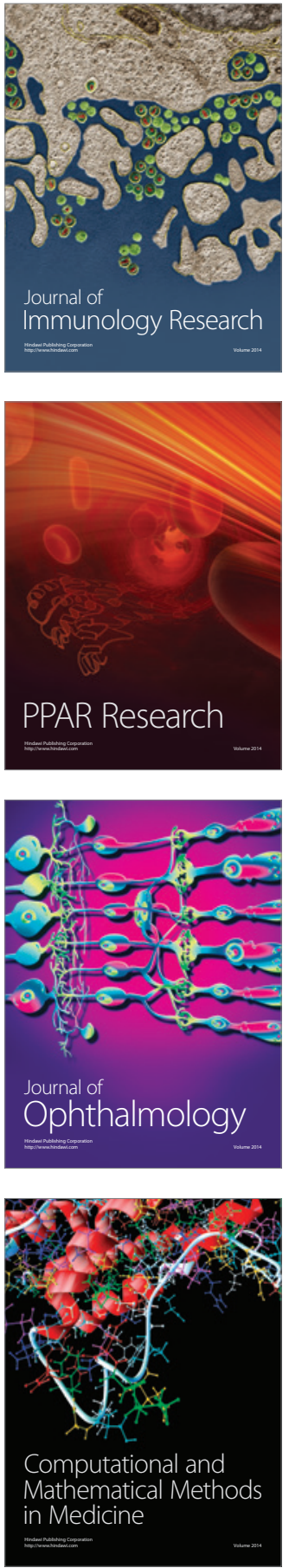

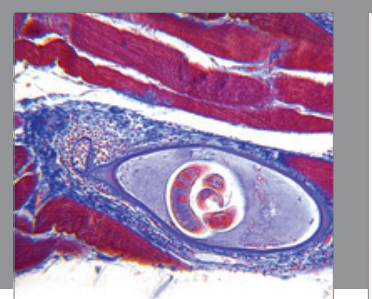

Gastroenterology Research and Practice

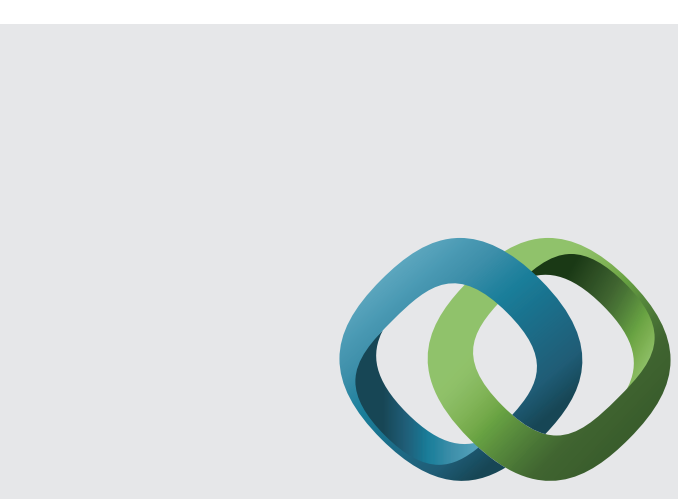

\section{Hindawi}

Submit your manuscripts at

http://www.hindawi.com
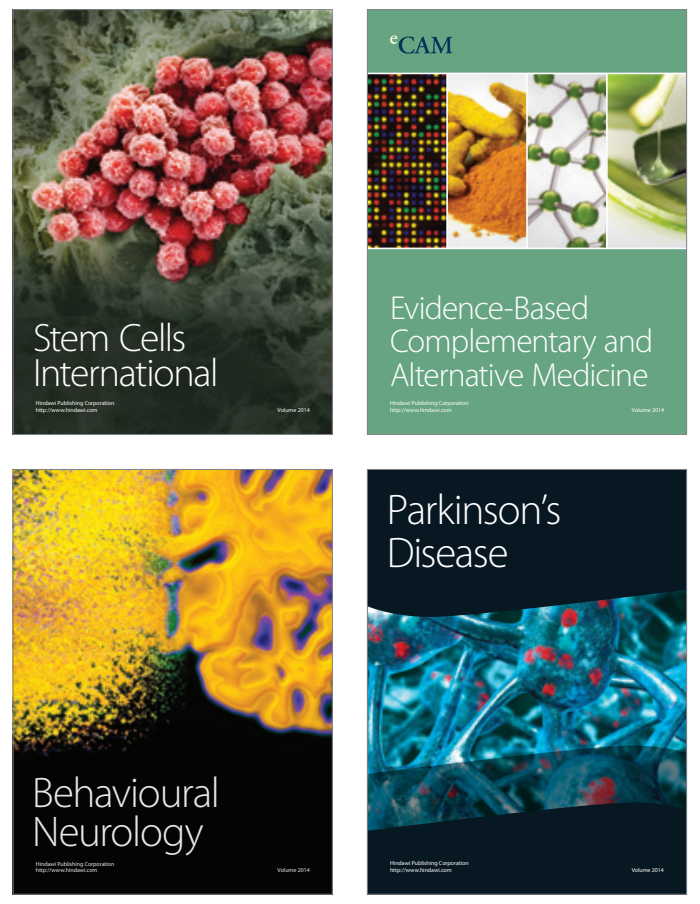
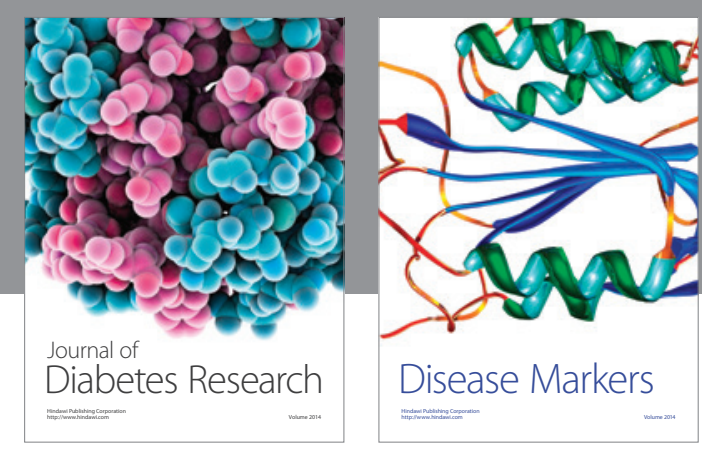

Disease Markers
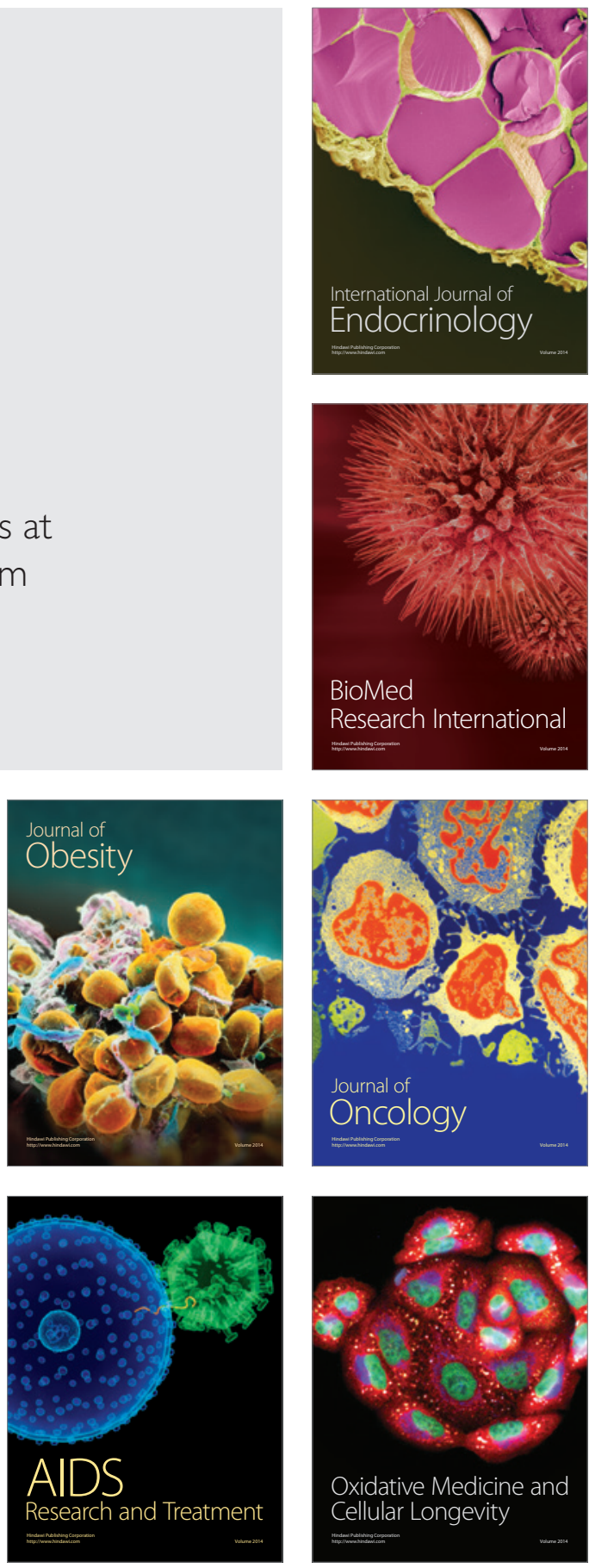